\title{
Vertical Splitting of Vortices in Geophysical Dipoles
}

\author{
Álvaro VIÚDEZ \\ Institut de Ciències del Mar, CSIC, Barcelona, Spain
}

(Manuscript received 18 December 2009, in final form 9 April 2010)

\begin{abstract}
The processes involved in the vertical splitting of vortices in geophysical dipoles, rotating and stably stratified, are investigated using a three-dimensional numerical model under the $f$-plane and Boussinesq approximations. Vertical splitting in asymmetric dipoles is possible when the vortices have a similar amount of potential vorticity but significantly differ in vertical extent. One representative case of vertical splitting is analyzed, and it is found that prior to the splitting there is a shearing period characterized by vertical unalignment and loss of horizontal axisymmetrization. The splitting starts when the upper and lower parts of the deep vortex independently experience vertical alignment and horizontal axisymmetrization. Vertical splitting also involves vortex horizontal splitting in the intermediate layers, which might explain the vertical asymmetry found in some isolated subsurface vortices in the ocean interior.
\end{abstract}

\section{Introduction}

Geophysical flows, as those happening in the earth's oceans and atmosphere, are strongly affected by fluid rotation and density stratification. These flows frequently develop three-dimensional baroclinic vortices that, owing to the background rotation and stratification, remain stable during long (from several months up to two years) time periods. Some well-known examples are the Gulf Stream rings (e.g., Richardson 1980), subsurface eddies of Mediterranean Water (meddies) in the North Atlantic (Armi et al. 1989), Agulhas Current eddies (Lutjeharms et al. 1989), and vortex dipoles generated from instability of coastal currents such as the Leeuwin Current (Griffiths and Pearce 1985) and the California Current (Ikeda et al. 1984). In particular, geophysical vortex dipoles exhibit a large resistance to separation (dipole breaking up into two separated vortices) and to horizontal splitting (one of the vortices breaks up into several smaller vortices of the same vertical extent). However, separation or horizontal splitting may occur when dipoles interact with topography (Centurioni 2002; Sutyrin et al. 2008; Hinds et al.

Corresponding author address: Álvaro Viúdez, Institut de Ciències del Mar, Passeig Marítim de la Barceloneta 37-49, 08003 Barcelona, Spain.

E-mail: aviudez@cmima.csic.es
2009) or with larger vortices (Dritschel and McIntyre 2008).

This study may be considered as part of the investigation on uneven vortex interactions. For example, Özuğurlu et al. (2008) considered interaction between two quasigeostrophic vortices of unequal potential vorticity (PV), whereas here we focus on unequal vertical size of ellipsoidal vortex cores. In particular, we investigate the processes involved in the vertical splitting of baroclinic dipoles, that is, when one of the vortices breaks up into several smaller vortices located in different vertical layers. To the best of our knowledge this vertical splitting has not been examined before.

Vertical splitting in baroclinic dipoles is possible when the vortices have a similar amount of volume integral of PV anomaly, but one of them (the splitting vortex) is notably deeper (has larger volume) than its vortex partner. This implies that the deep vortex has an absolute value of PV density anomaly smaller than its companion vortex. In this dipole configuration, the shallow vortex and the upper part of the deep vortex form a coherent dipole that acquires a large drift speed. Simultaneously, the lower part of the deep vortex, lacking most of the torque exerted by its companion vortex, slows down and therefore is left behind the shallow dipole. The deep vortex therefore suffers large vertical shear and eventually splits vertically. Our aim here is to address the transient processes occurring during this vertical splitting, and to do so we analyze a particular 
representative case. We do not intend to find the range in the parameter space, defined by the geometry and PV distribution of the vortices, in which vertical splitting occurs. This latter objective would require a very large number of numerical simulations and is outside the scope of this work. We do not intend either to simulate the baroclinic instability or other flow instability that may put two counterrotating vortices of unequal vertical size together. It is of course possible to numerically simulate this baroclinic instability from the beginning (see, e.g., Chérubin et al. 2007) and afterward analyze the resulting vertical splitting of vortices, but this would take much longer time integrations.

The vertical splitting of vortices is a transient threedimensional process, and, therefore, it becomes very difficult to be observed experimentally with the contemporary means of observation. We are not aware of experimental evidence of this process. However, this phenomenon may happen in some particular places: for example, in the Alboran Sea, east of the Strait of Gibraltar, where fast (large PV) shallow vortices of Atlantic water (the so-called Alboran gyres; Viúdez et al. 1996) interact with slow deep currents and vortices of Mediterranean Water. In fact, isolated subsurface vortices of Mediterranean Water and of unknown origin have been found in the northern Alboran Sea (Viúdez et al. 1998). Vertical splitting is also likely to happen when two currents (not necessarily vortices) of different vertical extent meet.

The basic definitions, theoretical basis of the numerical model, numerical parameters, and initial conditions are briefly given in section 2 . The numerical results are described, in a qualitative and quantitative way, in section 3. The splitting process involves vertical shearing, vertical alignment, and horizontal axisymmetrization of the vortices, which are analyzed in terms of the PV centers and PV intercentroid distances. In the dipole, the shear exerted by the shallow companion vortex over the deepest part of the deep splitting vortex diminishes as the upper pair of vortices propagates away as a dipole. Thus, the behavior of the baroclinic vortex in the dipole during splitting differs from that of an isolated vortex in background vertical shear flow. Finally, concluding remarks are given in section 4 .

\section{Numerical preliminaries}

\section{a. Basic definitions and theoretical basis of the numerical model}

We use a three-dimensional numerical model to simulate rotating, volume-preserving, nonhydrostatic, stably stratified flows under the $f$-plane and Boussinesq approximations (Dritschel and Viúdez 2003). The main characteristics of this model are the explicit conservation of PV via its advection on isopycnal surfaces using the contour advection algorithm of Dritschel and Ambaum (1997) and the prognostic equation is the material rate of change of ageostrophic horizontal vorticity (defined below). This approach allows long time integrations of vortical flows with minimum diffusive effects. The numerical simulations are initialized using the PV initialization approach (Viúdez and Dritschel 2003), which largely suppresses the generation of inertia-gravity waves due to unbalanced initial conditions.

The state variables are the components of the vector potential $\boldsymbol{\varphi}=(\varphi, \psi, \phi)$, which provide the velocity $\boldsymbol{u}=$ $-f \nabla \times \varphi$ and the vertical displacement of isopycnals $\mathcal{D}=$ $-\varepsilon^{2} \nabla \cdot \varphi$, where the Prandtl ratio $\varepsilon^{-1} \equiv c=N / f$ is the ratio between background Brunt-Väisälä and Coriolis frequencies. There are three prognostic scalar equations. The first two equations are for the rate of change of the dimensionless ageostrophic horizontal vorticity, $\boldsymbol{A}_{h}=$ $(\mathcal{A}, \mathcal{B}) \equiv\left(\boldsymbol{\omega}_{h}-\boldsymbol{\omega}_{h}^{g}\right) / f$, where $\boldsymbol{\omega}_{h}$ is the horizontal relative vorticity and $\boldsymbol{\omega}_{h}^{g}$ is the horizontal geostrophic vorticity. The third equation is the material conservation of PV anomaly $\varpi$ on isopycnals, $d \varpi / d t=0$, where the PV anomaly is defined by

$$
\varpi \equiv \Pi-1 \equiv \frac{\boldsymbol{\omega}+f \mathbf{k}}{f} \cdot \nabla d=\frac{\zeta}{f}-\frac{\partial \mathcal{D}}{\partial z}-\frac{\boldsymbol{\omega}}{f} \cdot \nabla \mathcal{D} ;
$$

$\Pi$ is the dimensionless PV, and $\boldsymbol{\omega}=(\xi, \eta, \zeta)$ is the relative vorticity vector. The value $d(\mathbf{x}, t) \equiv\left(\rho(\mathbf{x}, t)-\rho_{0}\right) /$ $\varrho_{0}$ represents the vertical location that the isopycnal located at $\mathbf{x}$ at time $t$ has in the reference density configuration defined by $\rho_{0}+\varrho_{0} z$, where $\rho(\mathbf{x}, t)$ is the density field; $\rho_{0}$ and $\varrho_{0}$ are a constant density and a constant density stratification, respectively; and $\mathcal{D}(\mathbf{x}, t) \equiv z-d(\mathbf{x}, t)$. Note that $N$ is a constant background Brunt-Väisälä frequency introduced for convenience in the mathematical formulation. Density stratification, of course, is not constant. The squared total Brunt-Väisälä frequency is given by

$$
\mathcal{N}^{2}(\mathbf{x}, t)=N^{2}\left(1-\frac{\partial \mathcal{D}}{\partial z}(\mathbf{x}, t)\right) .
$$

The horizontal potential $\varphi_{h} \equiv(\varphi, \psi)$ is obtained every time step by inverting $\boldsymbol{A}_{h}=\nabla^{2} \boldsymbol{\varphi}_{h}$, while the vertical potential $\phi$ is recovered from the inversion of the $\varpi$ definition (1).

\section{b. Numerical parameters}

We use a triply periodic numerical domain with $\left(n_{x}, n_{y}\right.$, $\left.n_{z}\right)=(128,128,128)$ grid points, vertical extent $L_{z}=2 \pi$ 

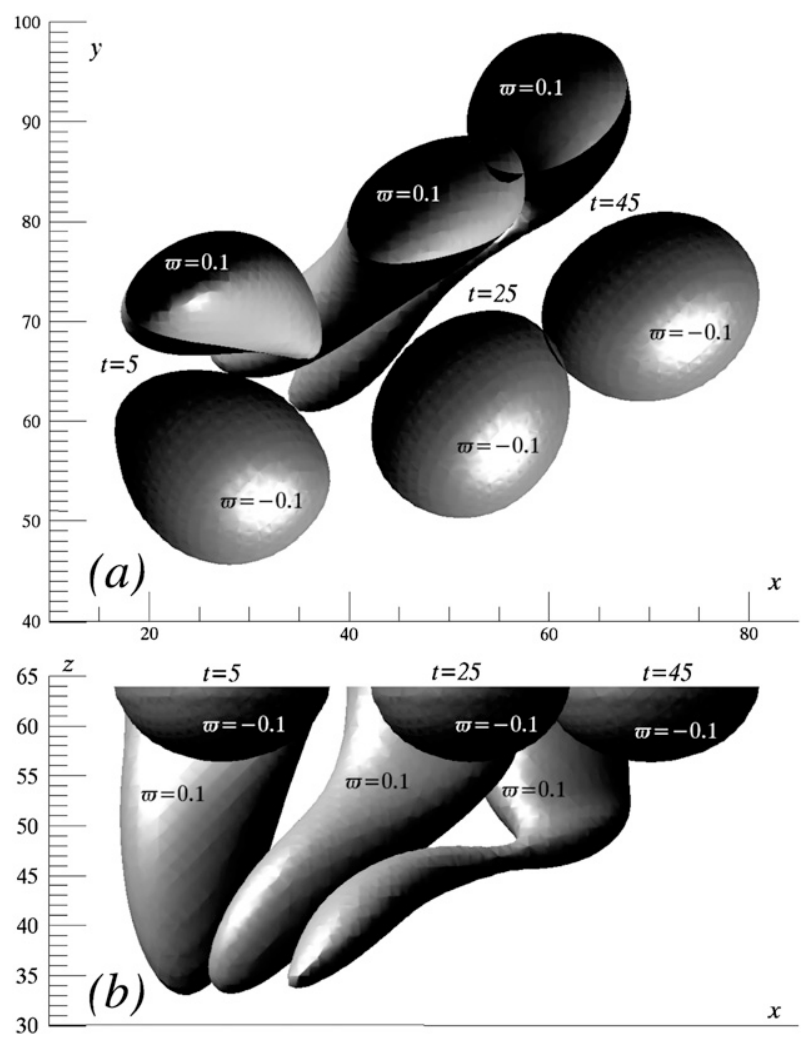

FIG. 1. Isosurfaces of $\varpi(x, y, z)= \pm 0.1$ at times $t=5,25$, and $45 T_{\mathrm{ip}}$. The views are (a) from the top and (b) from the south. In this figure and Fig. 2, a simple three-dimensional three-point boxcar filter was used to remove small-scale features and facilitate the visualization of the relevant larger-scale surfaces. Note the horizontal displacement of the dipole and the large speed of displacement of the upper part of the cyclone $(\varpi>0)$ compared to the lower part. The axes labels indicate distance in grid points and the axes scaling is isotropic in the QG space.

(which defines the unit of length), and horizontal extents $L_{x}=L_{y}=c L_{z}$ with a Prandtl ratio $c=N / f=10$. The quasigeostrophic $(\mathrm{QG})$ space scaling has extents $\left(L_{x} / c\right.$, $\left.L_{y} / c, L_{z}\right)$. We take the buoyancy period $T_{\mathrm{bp}}$ as the unit of time by setting $N=2 \pi$ so that one inertial period $T_{\mathrm{ip}}$ equals 10 buoyancy periods $\left(T_{\mathrm{ip}}=c T_{\mathrm{bp}}\right)$. The number of isopycnal surfaces $n_{l}$ is equal to the number of grid points in any direction, $n_{l}=128$. The time step $\delta t=0.01 T_{\mathrm{bp}}$, and the initialization time $\Delta t_{i}=5 T_{\mathrm{ip}}$. The initialization time is the minimum time interval required for the fluid to reach its prescribed initial state with minimal generation of inertia-gravity waves in the PV initialization approach (Viúdez and Dritschel 2003).

\section{c. Initial conditions}

The initial dipole consists of two ellipsoids of oppositely signed PV anomaly $\varpi$. The $\varpi$ is constant on ellipsoidal surfaces and varies linearly with the ellipsoidal volume with $\varpi=0$ on the outermost surface and $\varpi=\varpi_{0}^{ \pm}$at the
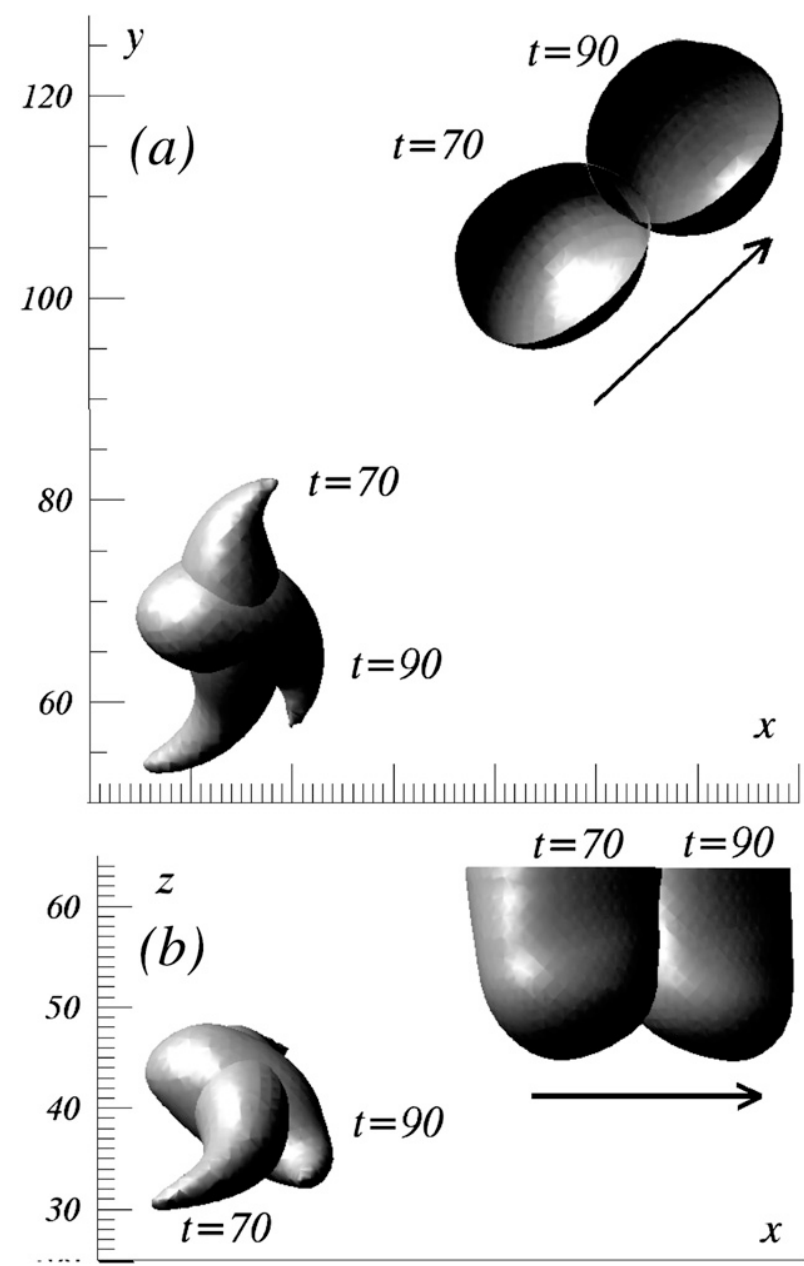

FIG. 2. Isosurfaces of $\varpi(x, y, z)=0.1$ (cyclone), as in Fig. 1, but at $t=70 T_{\mathrm{ip}}$ and $t=90 T_{\mathrm{ip}}$. The arrow indicates the direction of the dipole displacement. Note the precession of the lower cyclone around its PV center.

vortex core. Superscripts + and - refer to the cyclone and anticyclone, respectively. The $\varpi$ jump is fixed for all contours $\delta \varpi=\left|\varpi^{ \pm}\right| / n_{c}$ with $n_{c}=20$. In the particular case described here in detail, the outermost $\varpi$ ellipsoidal layer has horizontal major and minor semiaxes $\left(a_{x}^{ \pm}, a_{y}^{ \pm}\right)=$ $(0.6,0.4) c$ and vertical semiaxes $\left(a_{z}^{-}, a_{z}^{+}\right)=(0.27,2)$. We set $a_{x}^{ \pm}>a_{y}^{ \pm}$to facilitate the initial transition of the dipole toward a quasi-steady translating state in where the geometry of the steadily moving vortices is closer to an elliptical geometry than to a circular geometry (where $a_{x}^{ \pm}=a_{y}^{ \pm}$). Note the large vertical extent of the cyclone, $a_{z}^{+} \gg a_{z}^{-}$. On the other hand, the ratio of vertical to horizontal size of the anticyclone is typical of quasigeostrophic vortices, which is around 0.8 (Reinaud et al. 2003). It must be noted that the initial vertical extents of the PV ellipsoids are defined in the configuration, flat isopycnals space. During the initialization time the 

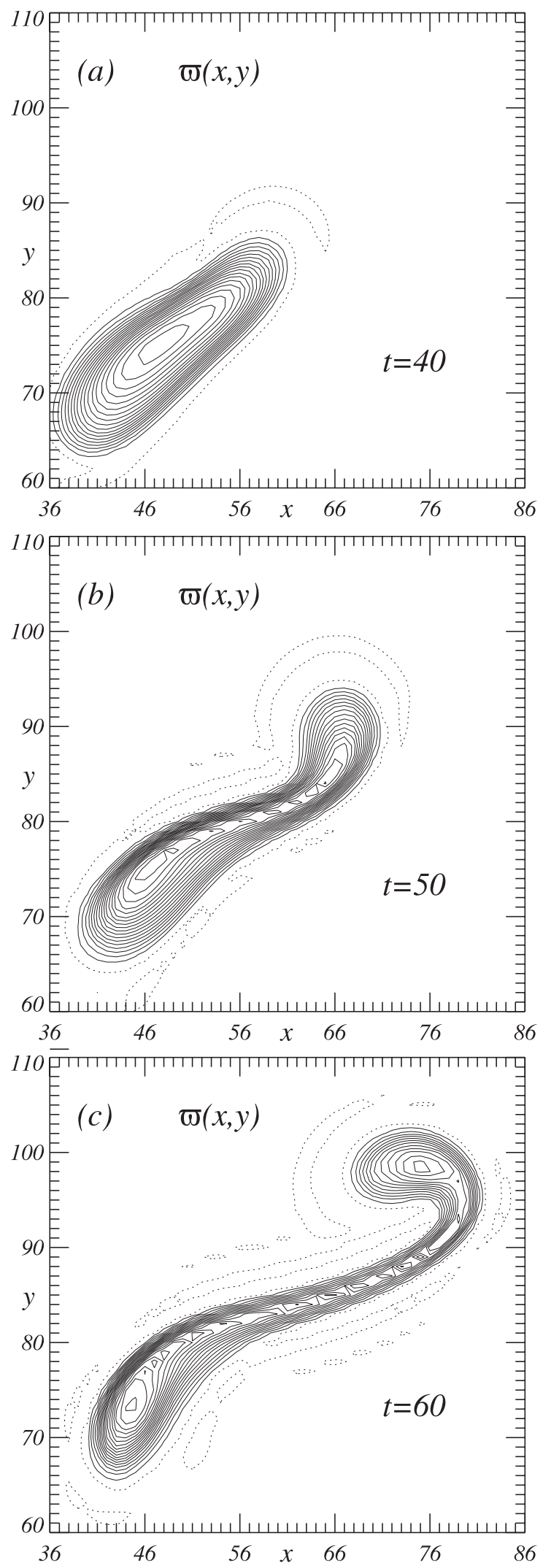

isopycnals of the anticyclone deepen owing to geostrophic balance making $a_{z}$ closer to 0.4 at the end of the initialization period. The initial distance between vortex centers is $2 a_{y}^{+}+0.01 c$. Further details on the PV configuration of an ellipsoid are given in Viúdez and Dritschel (2003). Note that, since the $\varpi$ field is symmetric with respect to the plane $z=0$, the numerical results apply to subsurface eddies (e.g, meddies) or, if the upper half is discarded, to surface eddies under the rigid lid approximation. Cyclones above anticyclones or heton-type vortices are not considered in this study.

Three-dimensional views of the PV configuration at the end of the initialization time $t=5 T_{\mathrm{ip}}$ and later times $\left(t=25\right.$ and $\left.45 T_{\mathrm{ip}}\right)$ are shown in Fig. 1. We set the PV anomaly extrema, $\left(\varpi_{0}^{-}, \varpi_{0}^{+}\right)=(-0.75,0.2)$, so that the vortex with the lowest absolute value of extreme PV density (the cyclone, with $\left|\varpi_{0}^{+}\right|<\left|\varpi_{0}^{-}\right|$) is the deepest vortex $\left(a_{z}^{+}>a_{z}^{-}\right.$and hence the volume $\left.\mathcal{V}^{+}>\mathcal{V}^{-}\right)$. However, the ratio between the amount of PV,

$$
\Pi_{\mathcal{V}} \equiv \int_{\mathcal{V}} \varpi(\mathbf{x}, t) d \mathcal{V}
$$

between vortices is $\Pi_{\mathcal{V}^{+}} / \Pi_{\mathcal{V}^{-}} \simeq-1.35$ so that the cyclone, though having a PV density extreme significantly smaller than the anticyclone, has a similar, though larger, amount of PV.

\section{Numerical results}

Since the $\varpi$ field is symmetric with respect to the plane $z=0$, only distributions in the lower half of the domain $\left(z \in[-\pi, 0]\right.$, vertical grid index $\left.i_{z} \in[0,64]\right)$ are displayed in the figures. During the initial time evolution of the dipole (Fig. 1) the anticyclone and the upper part of the cyclone advance side by side as a dipole, while the lower part of the cyclone, located away from the effect of the negative PV, is left behind. Thus, the cyclone experiences large vertical shearing but, on the other hand, has a remarkable initial resistance to splitting. Their PV surfaces endure $\pi / 4$ angle slopes (in the QG space) before splitting (Fig. 1b, $t=25 T_{\text {ip }}$ ). Such a resistance of geophysical vortices to vertical shear has been demonstrated using two-layer QG numerical models (Marshall and Parthasarathy 1993; Vandermeirsh et al. 2002).

FIG. 3. Horizontal distributions of $\varpi(x, y)$ on vertical level $i_{z}=$ $46(z=-0.88)$ at (a) $t=40 T_{\mathrm{ip}}$, (b) $t=50 T_{\mathrm{ip}}$, and (c) $t=60 T_{\mathrm{ip}}$. Contour interval $\Delta=0.01$. The cyclone experiences horizontal splitting on these intermediate layers. 

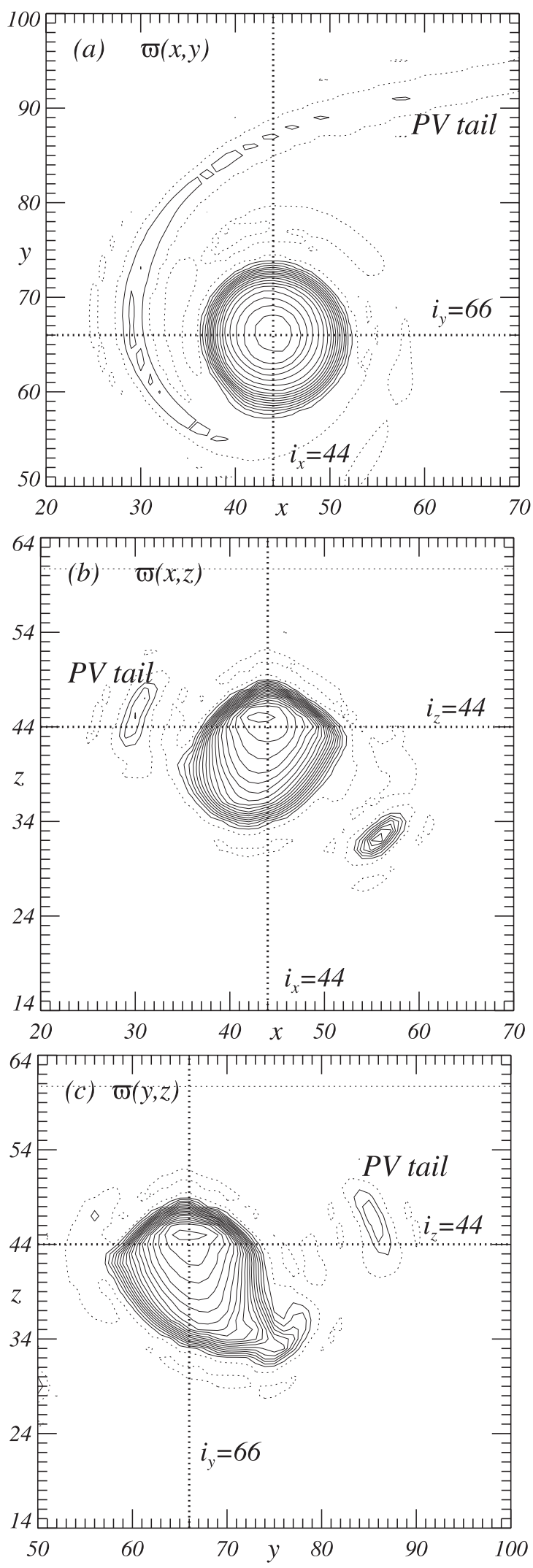

Once the cyclone can no longer undergo the large vertical tilting caused by the horizontal propagation of the upper dipole, the splitting process starts with the development of vertical alignment and horizontal axisymmetrization in the upper and lower parts, independently, of the cyclone. As discussed by Viera (1995), vertical alignment of the vortex is the extent to which the vortex axis (in this case, the line joining the PV centroids of all horizontal cross sections) becomes close to a vertical straight line. Horizontal axisymmetrization of the vortex is the extent to which the boundaries (in our case, the vortex PV contours) of a horizontal cross section at a given vertical level reach a circular shape. Vertical alignment of the upper and lower cyclones is observed in Figs. 1 and 2. Vertical alignment occurs in the upper cyclone faster than in the lower cyclone (Fig. 2b) due to the flow induced in the upper layers by the negative PV of the anticyclone.

Remarkably, the cyclone vertical splitting also involves vortex horizontal splitting at intermediate layers as evidenced from the time series of the horizontal distribution of $\varpi$ at the vertical level of splitting (the splitting layers, Fig. 3). This horizontal PV splitting assures that the vertical PV gradients at the boundary isopycnals after splitting remain small, so there is a smooth vertical PV transition across these isopycnals.

After splitting the lower cyclone precesses around its PV center as it experiences vertical alignment and horizontal axisymmetrization (Fig. 2a). This isolated vortex reaches a closely circular PV distribution in horizontal cross sections at long times (e.g., $t=120 T_{\text {ip }}$; Fig. 4a), though it is not vertically symmetric (Figs. $4 b, c$ ) since the upper PV surfaces are flatter than the lower ones. This is a consequence of PV material conservation and the PV horizontal splitting mentioned above (Fig. 3), which causes the amount of PV in the splitting layers be distributed among the upper and lower parts of the cyclone. A similar vertical asymmetry has been experimentally observed in the upper half layers of subsurface Mediterranean anticyclonic eddies (Armi et al. 1989; Paillet et al. 2002), where isopycnals look flatter than the ones of the lower half. This asymmetry might be related to the

FIG. 4. Distributions of $\varpi$ in the lower cyclone at $t=120 T_{\mathrm{ip}}$ on different planes: (a) $\varpi(x, y)$ on the horizontal plane $i_{z}=44$, (b) $\varpi(x, z)$ on the vertical plane $i_{y}=66$, and (c) $\varpi(y, z)$ on the vertical plane $i_{x}=44$. The long and thin PV tail still joins the upper and lower cyclones. The location of the horizontal and vertical planes is indicated. Contour interval $\Delta=0.01$. Note the flattening of $\varpi$ contours in the upper part of the cyclone in (b) and (c). 

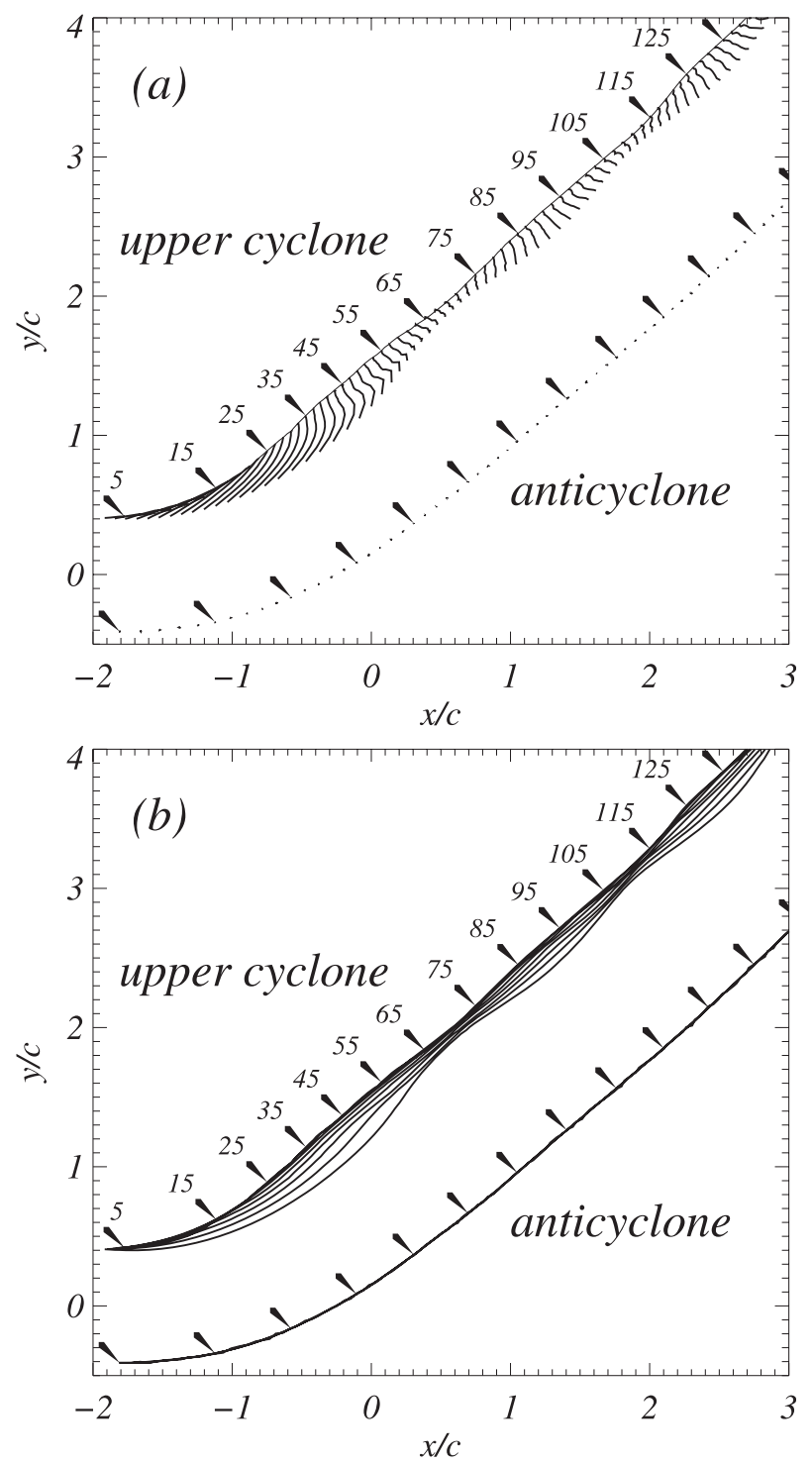

FIG. 5. Time series of the horizontal location of the PV centers $\mathbf{X}^{ \pm}(z, t)$. (a) The long continuous line is the upper cyclone trajectory at $z=0\left(i_{z}=64\right)$. The short lines starting along the upper cyclone trajectory join the horizontal locations of the PV centers at different depths $\mathbf{X}^{+}\left(z\left(i_{z}\right), t\right)$, for $i_{z} \in[49,64]$, every $2 T_{\mathrm{ip}}$. Ticks mark time in $T_{\mathrm{ip}}$. Similar lines are included in the anticyclone $\left(i_{z} \in[57\right.$, 64]), but these are so short that they appear only as points. (b) Vortex trajectories $\mathbf{X}^{ \pm}(z, t)$ at the depths given in (a). The anticyclone trajectories at different depths are so close in the figure that they appear as a single curve.

horizontal PV splitting described above and therefore be an indication of the way meddies separate from the Mediterranean undercurrent flowing along the Iberian continental slope, which sometimes involves a cyclonic partner.

To quantitatively characterize vertical alignment and horizontal axisymmetrization and, hence, vortex splitting,

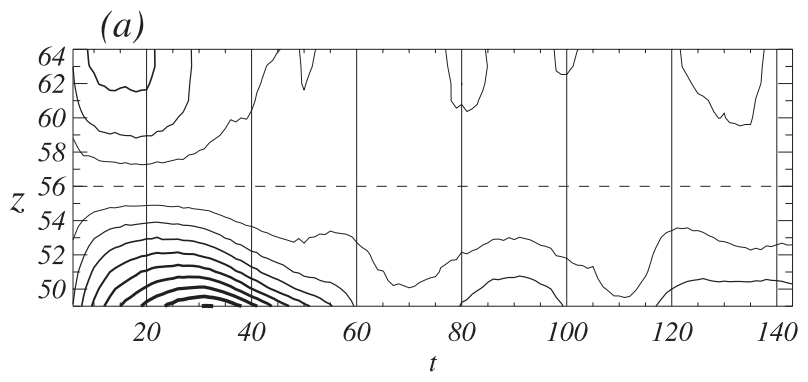

(b)

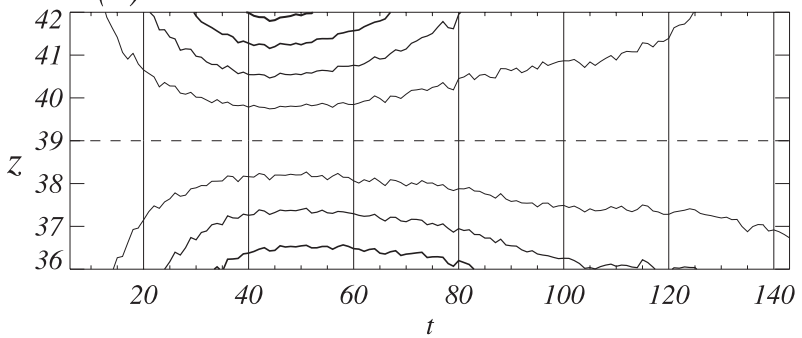

FIG. 6. Contours of the intercentroid distance $R(z, t)$ for (a) the upper cyclone (referenced to $i_{z r}=56$ ) and (b) the lower cyclone $\left(i_{z r}=39\right)$. Contour interval $\Delta=0.05 c$. Note the increase of $R$ during the shearing period and its decrease during the splitting period.

we define the amount of $\varpi$ in a given two-dimensional horizontal surface $\mathcal{S}$ as

$$
\Pi_{\mathcal{S}}(z, t) \equiv \int_{\mathcal{S}} \varpi\left(\boldsymbol{x}_{h}, z, t\right) d \mathcal{S} .
$$

Using (4), the vortices PV center at depth $z$ is

$$
\mathbf{X}^{ \pm}(z, t) \equiv \Pi_{\mathcal{S}^{ \pm}}^{-1}(z, t) \int_{\mathcal{S}^{ \pm}} \varpi\left(\mathbf{x}_{h}, z, t\right) \mathbf{x}_{h} d \mathcal{S} .
$$

Above $\mathcal{S}^{ \pm}$are the vortex surfaces where $\pm \varpi(\boldsymbol{x}, t) \geq 0.1$ and $\boldsymbol{x}_{h}=(x, y)$. The horizontal intercentroid distance (referenced to depth $z_{r}$ ) is defined as

$$
R(z, t) \equiv\left|\mathbf{X}(z, t)-\mathbf{X}\left(z_{r}, t\right)\right| .
$$

The time series of $\mathbf{X}^{ \pm}(z, t)$ (Fig. 5) and contours of $R(z, t)$ (Fig. 6a) show 1) the large vertical tilting of the lower part of the upper cyclone during, approximately, the first $30 T_{\mathrm{ip}}$, 2) the vertical alignment during splitting (decreasing of $R$ in the time period $\sim[30,55] T_{\mathrm{ip}}$ ), 3 ) bending with depth of the upper cyclone vertical axis toward the anticyclone (the short lines on the upper cyclone trajectory point to the anticyclone trajectory in Fig. 5a), and 4) cyclone axis oscillations after splitting. These oscillations are typical in geophysical vortices and are associated to vortex Rossby waves caused by the horizontal PV gradients in the vortices (Brunet and Montgomery 2002). 

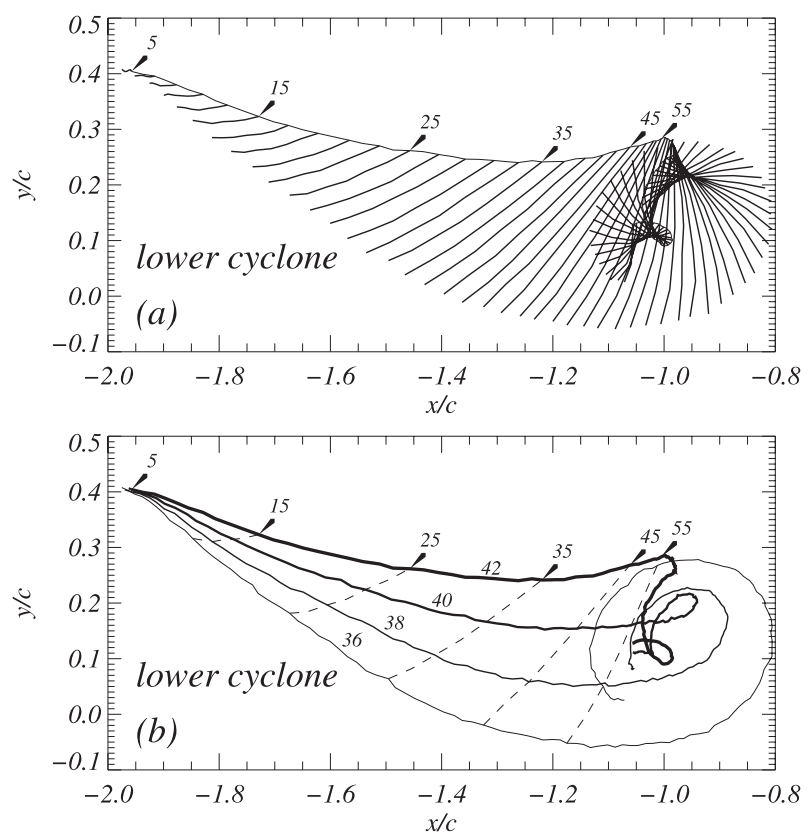

FIG. 7. As in Fig. 5, but for the lower cyclone. In (b) the vertical index $i_{z}=(36,38,40,42)$ of the cyclone trajectory $\mathbf{X}\left(i_{z}, t\right)$ is included, and some intercentroid lines (discontinuous lines) shown in (a) are repeated here for reference. The horizontal scales are isotropic. Note the two precession cycles of the vortex once its horizontal displacement decreases.

Contours of $R(z, t)$ show that vertical alignment occurs faster in the upper cyclone (which is part of the translating dipole; Fig. 6a) than in the lower cyclone (which is largely isolated from the other vortices; Fig. 6b). The lower cyclone (Fig. 7) experiences a short displacement during the shearing and splitting periods, after which starts precessing around its PV center.

The vortices velocity, speed, and direction angle are defined as

$$
\begin{aligned}
\boldsymbol{U}(z, t) & \equiv \frac{\partial \mathbf{X}}{\partial t}(z, t) ; \quad U \equiv\left|\left(U_{x}, U_{y}\right)\right|=\sqrt{U_{x}^{2}+U_{y}^{2}} ; \\
T & \equiv \arctan \frac{U_{y}}{U_{x}}
\end{aligned}
$$

(superscript \pm omitted). We also define the vertical average of $\chi$ between depths $z_{1}<z_{2}$ as

$$
\bar{\chi}(t) \equiv\left(z_{2}-z_{1}\right)^{-1} \int_{z_{1}}^{z_{2}} \chi(z, t) d z
$$

Contours of $U^{+}(z, t)$ in the upper cyclone (Fig. 8a) show the deceleration during the shearing period (from $t=0$ to $t \simeq 20 T_{\mathrm{ip}}$ ) as well as the acceleration and subsequent deceleration of the deeper PV tail during splitting (from
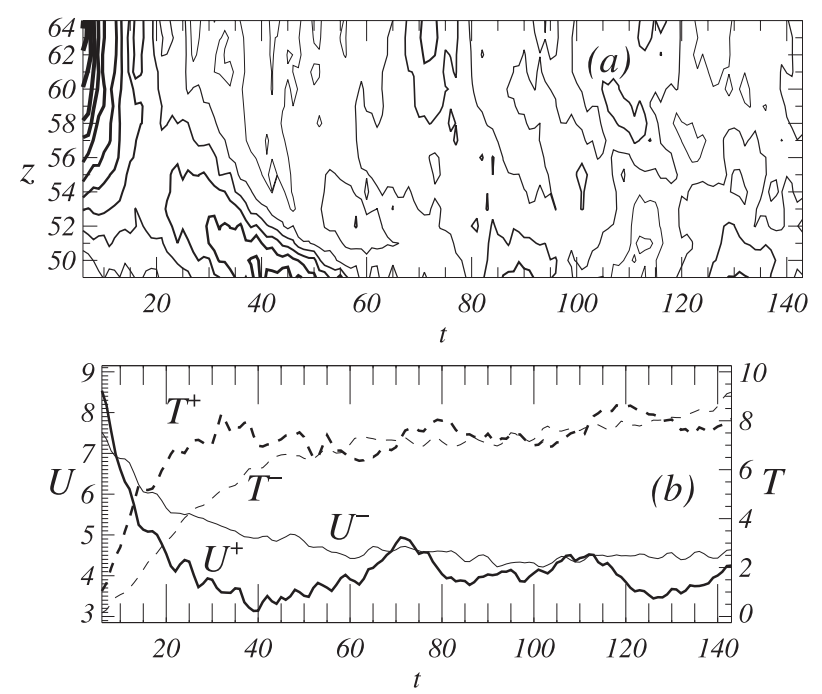

FIG. 8. (a) $U^{+}(z, t)-U_{\min }^{+}\left(U_{\min }^{+}=31 \times 10^{-3}, U^{+} \in[31,92] \times\right.$ $10^{-3}$, contour interval $\left.\Delta=5 \times 10^{-3}\right)$. (b) Time series of the vertically averaged vortices speed $\bar{U}^{ \pm}(t)\left(\times 10^{2}\right)$ and orientation $\bar{T}^{ \pm}(t)(\times 10)$ as defined by (8) with $z_{1}^{ \pm}=58$ and $z_{2}^{ \pm}=64$.

$t \simeq 20 T_{\mathrm{ip}}$ to $t \simeq 60 T_{\mathrm{ip}}$ ), which is associated to the vertical alignment of the upper cyclone. During the shearing period and in averaged terms (Fig. 8b), the deceleration of the upper cyclone, which is suffering large vertical tilting, is larger than the deceleration of the anticyclone. Vortex Rossby waves produce clear $40 T_{\mathrm{ip}}$ oscillations in $\bar{U}^{+}$after splitting, which also have a small effect in the upper cyclone direction $\bar{T}^{+}$. After splitting, the dipole trajectory has a small positive curvature [increase of $\bar{T}^{ \pm}(t)$, also seen in Fig. 5], consistent with $\bar{U}^{-}>\bar{U}^{+}$ (Fig. 8b) most of the time.

A linear measure of vertical alignment can be provided by fitting the paired data $\left(z_{i}, R\left(z_{i}, t\right)\right)$ to the linear model $\hat{R}(z, t)=\hat{a}(t)+\hat{b}(t) z$ using a least absolute deviation method. The angle of the vortex axis $\hat{T}^{+}(t) \equiv$ $\arctan \hat{b}^{+}(t)$ (Fig. 9) increases during the shearing period faster in the upper cyclone than in the lower cyclone. Splitting starts with vertical alignment at $t \simeq 31 T_{\text {ip }}$ in the upper cyclone and at $t \simeq 47 T_{\mathrm{ip}}$ in the lower cyclone. Clearly, vertical alignment is faster in the upper cyclone (with a rate $\Delta \hat{T}^{+} / \Delta t \simeq 2.7^{\circ} / 24 T_{\mathrm{ip}} \simeq 0.11^{\circ} / T_{\mathrm{ip}}$ ) than in the lower cyclone (rate $\Delta \hat{T}^{+} / \Delta t \simeq 2.2^{\circ} / 42 T_{\text {ip }} \simeq$ $0.046^{\circ} / T_{\text {ip }}$ ).

To quantitatively describe horizontal axisymmetrization, we introduce the distance relative to the PV centroids and PV anomaly data points in every vertical level,

$$
\begin{aligned}
r_{i}^{ \pm}(z, t) & \equiv\left|\boldsymbol{x}_{h i}^{ \pm}(z, t)-\boldsymbol{X}^{ \pm}(z, t)\right|, \\
\varpi_{i}^{ \pm}(z, t) & \equiv \varpi\left(\boldsymbol{x}_{h i}^{ \pm}, z, t\right), \quad i=1, \ldots, N^{ \pm}(z, t),
\end{aligned}
$$



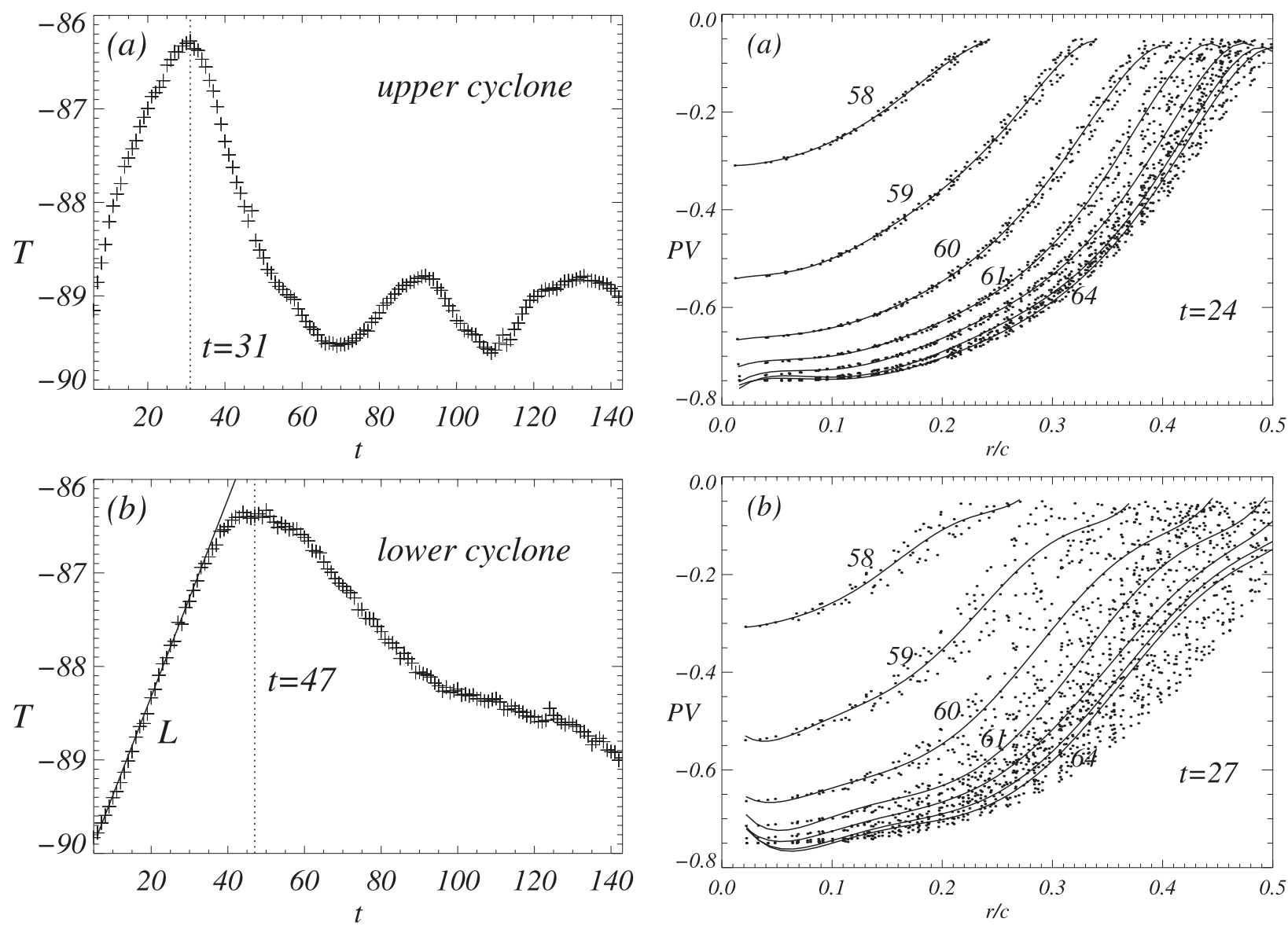

FIG. 9. Time series of $\hat{T}^{+}(t) \equiv \arctan \hat{b}^{+}(t)$ (in sexagesimal degrees) for (a) the upper cyclone $\left(z_{i} \in[49,57]\right)$ and $(b)$ the lower cyclone $\left(z_{i} \in[36,42]\right)$. Note the closely linear growth (line $L$ ) during the shearing period.

where $N^{ \pm}(z, t)$ is the number of grid points such that $\pm \varpi_{i}^{+}(z, t) \geq 0.05$. The pairs $\left(r_{i}(z, t), \varpi_{i}(z, t)\right)$ are then adjusted to a polynomial of degree $n=6$,

$$
\hat{\varpi}(z, t ; r)=\sum_{m=0}^{n} A_{m}(z, t) r^{m} .
$$

An example of the data $\left(r_{i}(z, t), \varpi_{i}(z, t)\right)$ and the polynomials $\hat{\omega}(z, t ; r)$ is given in Fig. 10 for the anticyclone at several vertical levels and two times. Using (10), we obtain a measure the PV deviations from a radial distribution, and therefore a measure of the lack of horizontal axisymmetrization, as the standard deviation

$$
\varpi^{\prime}(z, t) \equiv\left(\frac{1}{N(z, t)} \sum_{i=1}^{N(z, t)}\left(\varpi_{i}(z, t)-\hat{\varpi}\left(z, t ; r_{i}\right)\right)^{2}\right)^{1 / 2}
$$

FIG. 10. Scatterplots of $\left(\varpi_{i}^{-}\left(z, t_{0}\right), r_{i}^{-}\left(z, t_{0}\right)\right)$ and polynomials $\hat{\varpi}(z, t ; r)$ (continuous lines) at different depths $\left(i_{z} \in[38,64]\right.$ as indicated) and at (a) $t_{0}=24 T_{\mathrm{ip}}$ and (b) $t_{0}=27 T_{\mathrm{ip}}$. Note the scattering increase from (a) $t=24 T_{\text {ip }}$ to (b) $t=27 T_{\text {ip. }}$.

In the anticyclone (Fig. 11a) $\varpi^{\prime}(z, t)$ reaches absolute maxima during the shearing period and has an oscillatory behavior (with a periodicity of about $5.9 T_{\mathrm{ip}}$ ), consistent with the scattering increase from $t=24 T_{\mathrm{ip}}$ to $t=27 T_{\mathrm{ip}}$ shown in Fig. 10. These oscillations are the effect of vortex Rossby waves in the horizontal axisymmetrization that, due to the large horizontal gradients of PV and vortex vertical extent, differ from the vortex Rossby waves in the cyclone (which have a much larger period of about $40 T_{\text {ip }}$ ). In the upper cyclone (Fig. 11b) $\varpi^{\prime}(z, t)$ increases during the shearing period and decreases afterward. The small vertical tilt of $\varpi^{\prime}(z, t)$ maxima indicates that horizontal axisymmetrization depends slightly on the vertical layer. The time series of the vertical average of the lack of axisymmetrization $\left\langle\varpi^{\prime}\right\rangle(t)$ (Fig. 12) indicates that axisymmetrization decreases and increases $\left(\left\langle\varpi^{\prime}\right\rangle(t)\right.$ rises and falls) noticeably in the upper cyclone in the process of vertical splitting (during approximately the first $\left.40 T_{\text {ip }}\right)$. In the anticyclone $\left\langle\varpi^{\prime}\right\rangle(t)$ is dominated by the 
(a) anticylone

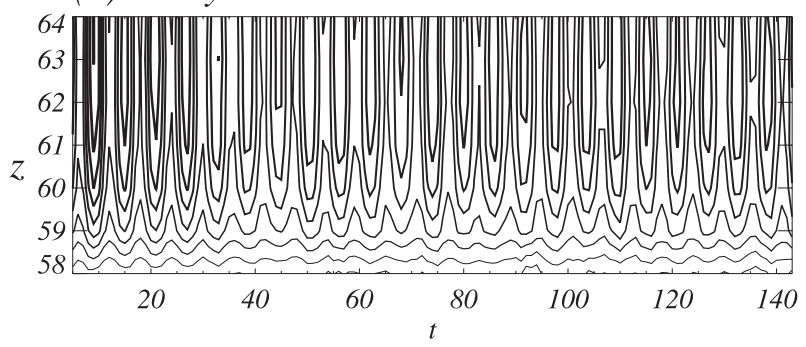

(b) upper cyclone

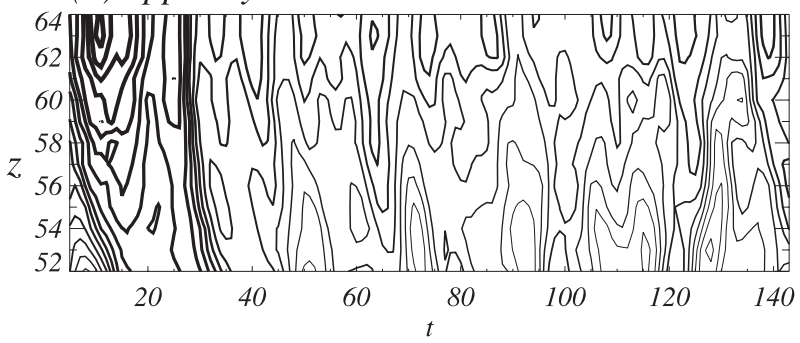

FIG. 11. $\varpi^{\prime}(z, t)-\varpi_{\min }^{\prime}$ in (a) the anticyclone $\left(\varpi^{\prime} \in[27,88] \times\right.$ $\left.10^{-3}, \Delta=5 \times 10^{-3}\right)$ and $(b)$ in the upper cyclone $\left(\varpi^{\prime} \in[15,38] \times\right.$ $\left.10^{-3}, \Delta=1.25 \times 10^{-3}\right)$. The time oscillations of $\varpi^{\prime}(z, t)$ in (a) are vortex Rossby waves with a periodicity of about $130 T_{\mathrm{ip}} / 22 \simeq 5.9 T_{\mathrm{ip}}$.

oscillations with a period $\simeq 5.9 T_{\text {ip }}$ associated to the vortex Rossby waves mentioned above.

Several numerical experiments with different initial dipole configurations (modifying $\varpi_{0}^{+}$and $a_{z}^{+}$) were carried out and verified the robustness of the previous results. When $\varpi_{0}^{+}$is increased, vortex splitting occurs, but the dipole acquires larger positive curvature. In these cases, the shearing process in the PV isosurfaces resembles a whiplike wave propagating down to the deep vortex. Vertical splitting is prevented when the cyclone depth decreases to about $a_{z}^{+}=0.8$. If $\varpi_{0}^{+}$increases too much, however, the shallow anticyclone may mostly spin around the cyclone and vertical splitting is also prevented. In some cases of strong curvature the lower vortex experiences large shearing and may break into several smaller vortices that axisymmetrize independently.

\section{Concluding remarks}

Very idealized numerical PV configurations show that vertical splitting of geophysical vortices is possible and most likely to happen in asymmetric dipoles when the vortices have a similar amount of PV but differ notably in their vertical extent. Prior to the splitting process there is a shearing period characterized by vertical unalignment and loss of horizontal axisymmetrization. The splitting process properly speaking starts when the upper and lower parts of the vortex experience vertical alignment and horizontal axisymmetrization, which may

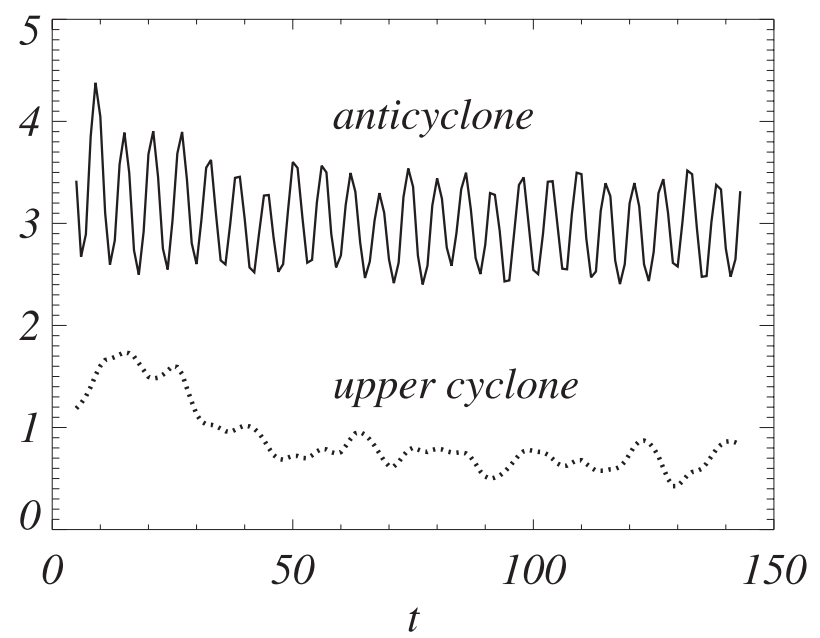

FIG. 12. Time series of the vertical average of the axisymmetrization $\left\langle\varpi^{\prime}\right\rangle(t) \equiv N^{-1} \sum_{i_{z 1}}^{i_{z N}} \varpi^{\prime}\left(i_{z}, t\right)\left(\times 10^{2}\right)$ for the anticyclone $(N=7$, $i_{z 1}=58$, and $\left.i_{z N}=64\right)$ and upper cyclone $\left(N=13, i_{z 1}=52\right.$, and $i_{z N}=$ 64). The oscillations in the anticyclone are due to the vortex Rossby waves shown in Fig. 11a.

occur at different rates in both parts of the splitting vortex. Vertical splitting also involves vortex horizontal splitting at the intermediate layers, which might explain the vertical asymmetry found in some isolated subsurface vortices in the ocean. This kind of unstable vertically asymmetric vortex dipoles may be generated in the ocean by the progressive approaching of two counterrotating isolated surface vortices, by instability of surface coastal currents such a the California Current or the Australian Leeuwin Current, or by instability of subsurface coastal currents such as the Mediterranean Current flowing along the Iberian continental slope. The vertical splitting of vortices in dipoles or similar PV configurations may provide an explanation for the existence of some isolated subsurface vortices in the ocean interior.

Acknowledgments. I thank two anonymous reviewers for the very helpful criticisms. Partial support for this research has come from the Spanish Ministerio de Ciencia e Innovación (Grant CTM2008-06438-C02-01/ MAR).

\section{REFERENCES}

Armi, L., D. Hebert, N. Oakey, J. F. Price, P. L. Richardson, H. T. Rossby, and B. Ruddick, 1989: Two years in the life of a Mediterranean salt lens. J. Phys. Oceanogr., 19, 354-370.

Brunet, G., and M. T. Montgomery, 2002: Vortex Rossby waves on smooth circular vortices: Part I. Theory. Dyn. Atmos. Oceans, 35, 153-177.

Centurioni, L. R., 2002: Dynamics of vortices on a uniformly shelving beach. J. Fluid Mech., 472, 211-228, doi:10.1017/ S0022112002002252. 
Chérubin, L., X. Carton, and D. G. Dristchel, 2007: Vortex dipole formation by baroclinic instability of boundary currents. J. Phys. Oceanogr., 37, 1661-1667.

Dritschel, D. G., and M. H. P. Ambaum, 1997: A contour-advective semi-Lagrangian algorithm for the simulation of fine-scale conservative fields. Quart. J. Roy. Meteor. Soc., 123, 1097-1130.

— , and A. Viúdez, 2003: A balanced approach to modelling rotating stably stratified geophysical flows. J. Fluid Mech., 488, 123-150, doi:10.1017/S0022112003004920.

_ , and M. E. McIntyre, 2008: Multiple jets as PV staircases: The Phillips effect and the resilience of eddy-transport barriers. J. Atmos. Sci., 65, 855-874.

Griffiths, R. W., and A. F. Pearce, 1985: Instability and eddy pairs on the Leeuwin Current south of Australia. Deep-Sea Res., 32, 1511-1534.

Hinds, A. K., I. Eames, E. R. Johnson, and N. R. McDonald, 2009: Laboratory study of vortex dipoles interacting with step topography. J. Geophys. Res., 114, C06006, doi:10.1029/2008JC005210.

Ikeda, M., L. A. Mysak, and W. J. Emery, 1984: Observation and modeling of satellite-sensed meanders and eddies off Vancouver Island. J. Phys. Oceanogr., 14, 3-21.

Lutjeharms, J. R. E., R. Catzel, and H. R. Valentine, 1989: Eddies and other boundary phenomena of the Agulhas Current. Cont. Shelf Res., 9, 597-616.

Marshall, J. S., and B. Parthasarathy, 1993: Tearing of an aligned vortex by a current difference in two-layer quasi-geostrophic flow. J. Fluid Mech., 255, 157-182, doi:10.1017/S0022112093002435.

Özuğurlu, E., J. N. Reinaud, and D. G. Dritschel, 2008: Interaction between two quasi-geostrophic vortices of unequal potential vorticity. J. Fluid Mech., 297, 395-414, doi:10.1017/ S0022112007000018.

Paillet, J., B. L. Cann, X. Carton, Y. Morel, and A. Serpette, 2002: Dynamics and evolution of a northern meddy. J. Phys. Oceanogr., 32, 55-79.

Reinaud, J. N., D. G. Dritschel, and C. R. Koudella, 2003: The shape of vortices in quasi-geostrophic turbulence. J. Fluid Mech., 474, 175-192, doi:10.1017/S0022112002002719.

Richardson, P. L., 1980: Gulf Stream ring trajectories. J. Phys. Oceanogr., 10, 90-104.

Sutyrin, G. G., X. Perrot, and X. Carton, 2008: Integrable motion of a vortex dipole in an axisymmetric flow. Phys. Lett., 372A, 5452-5457, doi:10.1016/j.physleta.2008.06.038.

Vandermeirsh, F., Y. Morel, and G. Sutyrin, 2002: Resistance of a coherent vortex to a vertical shear. J. Phys. Oceanogr., 32, 3089-3100.

Viera, F., 1995: On the alignment and axisymmetrization of a vertically tilted geostrophic vortex. J. Fluid Mech., 289, 29-50, doi:10.1017/S0022112095001224.

Viúdez, A., and D. G. Dritschel, 2003: Vertical velocity in mesoscale geophysical flows. J. Fluid Mech., 483, 199-223, doi:10.1017/ S0022112003004191.

— J. Tintoré, and R. L. Haney, 1996: Circulation in the Alboran Sea as determined by quasi-synoptic hydrographic observations. Part I. Three-dimensional structure of the two anticyclonic gyres. J. Phys. Oceanogr., 26, 684-705.

, J.-M. Pinot, and R. L. Haney, 1998: On the upper layer circulation in the Alboran Sea. J. Geophys. Res., 103, 21 65321666 